\title{
How action selection can be embodied: intracranial gamma band recording shows response competition during the Eriksen flankers test
}

\author{
Fausto Caruana ${ }^{1,2}$ *, Sebo Uithol ${ }^{2}$, Gaetano Cantalupo ${ }^{3}$, Ivana Sartori ${ }^{4}$, Giorgio Lo Russo ${ }^{4}$ and \\ Pietro Avanzini ${ }^{2,5}$ \\ ${ }^{1}$ Brain Center for Social and Motor Cognition - Istituto Italiano di Tecnologia, Parma, Italy \\ ${ }^{2}$ Department of Neuroscience, University of Parma, Parma, Italy \\ ${ }^{3}$ Department of Life and Reproduction Sciences, University of Verona, Verona, Italy \\ 4 "Claudio Munari" Center for Epilepsy Surgery, Ospedale Niguarda-Ca' Granda, Milan, Italy \\ ${ }^{5}$ Department of Biomedical, Metabolism, and Neural Science, NOCSAE Hospital, University of Modena and Reggio Emilia, Modena, Italy
}

\section{Edited by:}

Louise Barrett, University of

Lethbridge, Canada

\section{Reviewed by:}

Douglas O. Cheyne, Hospital for Sick Children, Canada

Matthew R. Longo, Birkbeck,

University of London, UK

\section{*Correspondence:}

Fausto Caruana, Brain Center for

Social and Motor Cognition - Istituto

Italiano di Tecnologia, Via Volturno 39,

43125 Parma, Italy

e-mail: fausto.caruana@unipr.it
Recent findings in monkeys suggest that action selection is based on a competition between various action options that are automatically planned by the motor system. Here we discuss data from intracranial EEG recordings in human premotor cortex (PMC) during a bimanual version of the Eriksen flankers test that suggest that the same principles apply to human action decisions. Recording sites in the dorsal PMC show an early but undifferentiated activation, a delayed response that depends on the experimental conditions and, finally, a movement related activation during action execution. Additionally, we found that the medial part of the PMC show a significant increase in response for ipsilateral trials, suggesting a role in inhibiting the wrong response. The ventral PMC seems to be involved in action execution, rather than action selection. Together these findings suggest that the human PMC is part of a network that specifies, selects, and executes actions.

Keywords: embodiment, action selection, intracerebral recording, human PMC

\section{INTRODUCTION}

In the cognitivist's framework, action decisions stem from a conveniently arranged system. The central cognitive module evaluates the input from the visual areas, selects a specific action, and subsequently informs the premotor cortex (PMC) for action planning and execution (Miller et al., 1960; see Fodor, 1983). There is abundant evidence that this now infamous "sandwich model" of cognition is empirically untenable (Uithol et al., 2012, 2014; Engel et al., 2013; Schurger and Uithol, in press), and very few scientists today would explicitly endorse such a view on cognition.

The sandwich model is by and large replaced with embodied approached to cognition. Within these approaches cognition is considered to be something that is not detached from perception and action, but something that emerges from the tight connection between these processes. However, these embodied approaches face a new problem with respect to action control: how can embodied processes result in a cognitive decision to perform a certain action? There is no central decision center that is planning the action, but at the same time it is highly unlikely that decisions are purely context driven sensorimotor links. How can action decisions be embodied and cognitive at the same time?

Electrophysiological recordings in monkeys gave rise to an interesting model that could explain how decisions can come about in an embodied cognitive system (Cisek, 2007; Cisek and Kalaska, 2010). In this "affordance competition hypothesis" the observation of a stimulus suggesting multiple competing actions elicits the parallel planning of all possible actions. Importantly, these action options are concrete action plans, specified for the appropriate context across fronto-parietal circuits. The options compete for further processing and are biased by input from prefrontal cortical regions and the basal ganglia. A decision in this framework is the prevalence of one option - through biasing influence of context features and internal processes - and the suppression of the others (see also Crammond and Kalaska, 1994; Gail et al., 2009). Recent evidence suggests that similar processes underlie human decision processes. Michelet et al. (2010) showed that the corticospinal excitability during simple decisions (i.e., a Eriksen flankers test) reflects a competition between action options. While these important findings suggest a similar mechanism at work in human action selection, they do not provide details on the source and dynamics of these selection processes.

Here we want to discuss the first evidence of a competition between action options using intracranial recordings in humans, and discuss the temporal dynamics underlying this process. We used a bimanual version of the classic Eriksen flankers test (Eriksen and Eriksen, 1974). This classic test consists in the presentation of a directional arrow, flanked by congruent, or incongruent, similar directional arrows, or by neutral stimuli. Subjects are asked to respond as fast as possible in a way that is congruent to the direction indicated by the central arrow, and independently by the flanked distractors. Behavioral studies showed that the response is delayed 
when the central cue is flanked by incongruent directional stimuli, and the increase in reaction time is considered to reflect the interference between competing responses. During this test we recorded high gamma-band activity $(50-150 \mathrm{~Hz})$ from six patients with frontal implantations involving the PMC. We found (1) an early but non-specific response to an observed stimulus (100 ms after stimulus onset) of PMC neurons, (2) a delayed activation, modulated by the experimental condition, and (3) a modulation depending on the subsequent action. Together, these findings suggest an action selection process in the motor system as suggested in the affordance competition hypothesis.

\section{MATERIALS AND METHODS PARTICIPANTS}

The experiment was performed on six patients (gender: $F=3$; $M=3$; age: $23 \pm 9$; implantation side: right $=1$; left $=5$ ) suffering from drug-resistant focal epilepsy and stereotactically implanted with intracerebral electrodes as part of their pre-surgical evaluation, at the "Claudio Munari" Center for Epilepsy Surgery, Ospedale Niguarda-Ca' Granda, Milan, Italy. Implantation sites were selected on purely clinical grounds, on the basis of seizure semiology, scalp-EEG, and neuroimaging studies, and with no reference to the present experimental protocol. Patients were fully informed of the electrode implantation and stereo-EEG recordings, and, according to the Declaration of Helsinki (BMJ 1991; 302: 1194) gave written informed consent to participate in the study. Experimental procedures were approved by the EthicalScientific Committee of the Ospedale Niguarda-Ca' Granda. We selected patients whose precentral region was not affected by epileptic activity. No seizures were recorded during the $24 \mathrm{~h}$ prior to the experiment. No alteration in the sleep/wake cycle was observed, and no additional pharmacological treatment was applied before the experiment. Patients did not show any motor or cognitive deficits during the examination; they fully understood the instructions and easily performed the experimental task.

\section{ELECTRODE IMPLANTATION}

For each patient, up to fifteen depth electrodes were implanted in different regions of the brain including the PMC. To reach the clinically relevant targets, the stereotactic coordinates of each electrode were calculated preoperatively based on the individual's cerebral MRI. Each electrode had a diameter of $0.8 \mathrm{~mm}$ and comprised 10-15 $2 \mathrm{~mm}$ long contacts, spaced $1.5 \mathrm{~mm}$ apart (DIXI ${ }^{\circledR}$, Besançon, France). Cerebral structures explored by each electrode contact were determined by coregistration of pre-implantation volumetric brain MRI with post-implantation volumetric brain CT, and visualized by a software package for visualization and image analysis (3DSlicer ${ }^{\circledR}$ ).

\section{PROCEDURE}

Recordings were obtained in a dimly light quiet room. The patient sat approximately $100 \mathrm{~cm}$ away from the laptop display where the stimuli were presented. The stimuli consisted in a horizontal array of three symbols consisting in a central arrow (target), directed toward right or left, flanked by identical arrows in the same (congruent) or opposite (incongruent) direction, or by squares composed by the same graphical elements of the target arrows, but without any directional information (neutral condition). Consequently six conditions were presented: right congruent (RC), right incongruent $(\mathrm{RI})$, right neutral $(\mathrm{RN})$, left congruent (LC), left incongruent (LI), and left neutral (LN). Each stimulus was presented in random order for $200 \mathrm{~ms}$ after a fixation cross of $2000 \mathrm{~ms}$ (see Figure 1, upper panel). Each condition was presented
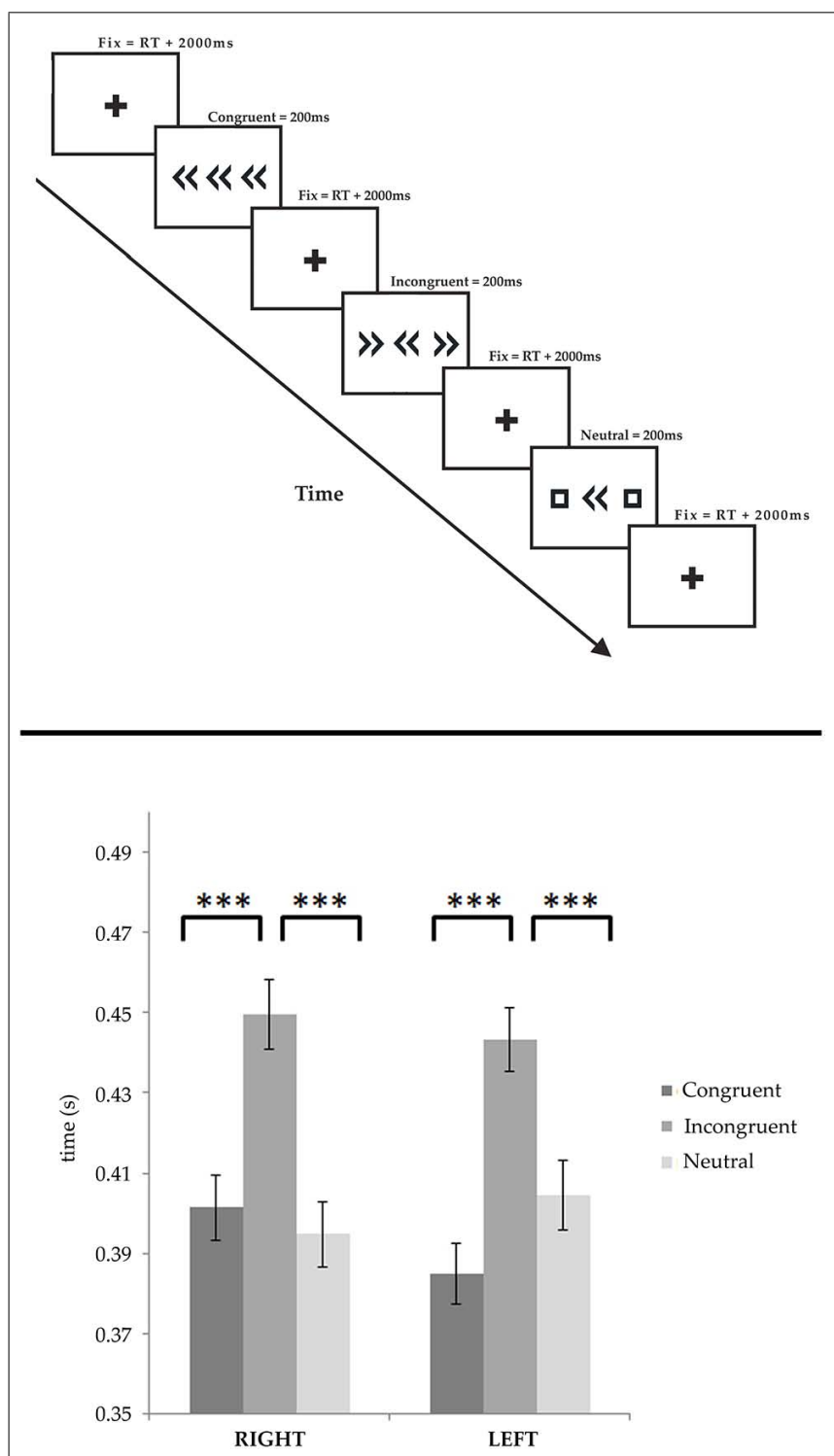

FIGURE 1 | (Upper panel) Experimental paradigm. The stimuli consisted in a horizontal array of three symbols consisting in a central arrow (target), flanked by identical arrows in the same (congruent) or opposite (incongruent) direction, or by squares composed by the same graphical elements of the target arrows, but without any directional information (neutral condition). Each stimulus was presented for $200 \mathrm{~ms}$ and followed by a fixation cross lasting the response time plus two additional seconds. (Lower panel) Behavioral results are shown for both the left and right responses, separately. In both cases the incongruent trials elicited significantly delayed responses, as compared to the congruent and neutral trials. No condition showed any significant effect of side. *** $p<0.0001$. 
60 times. During the task the hands of the patients leaned on the keyboard, with the index fingers on the two buttons located at the top right and top left side of the keyboard (the "Esc" and "Del" button). The patient was asked to press the correct button (the button congruently to the central target) as fast as possible using the corresponding hand. A short practice session $(<2 \mathrm{~min})$ took place before the start.

\section{STEREO-EEG RECORDING AND ANALYSIS}

During the experiment continuous stereo EEG (sEEG) was recorded with a $1000 \mathrm{~Hz}$ sampling rate by means of a 192 channelEEG device (EEG-1200 Neurofax, Nihon Kohden ${ }^{\circledR}$ ). Each channel was referred to a contact in the white matter far from the recording sites, in which low and high frequency electrical stimulations did not produce any subjective or objective manifestation (neutral reference). At the end of each experimental session, sEEG data were exported and the activity of each contact located in the precentral region ( $n=46$ recording sites) was selected. A visual inspection was carried out by clinicians in order to ensure the absence of any pathological interictal activity. Trials showing artifacts were removed. A band-pass filter $(0.015-500 \mathrm{~Hz})$ was applied to avoid any aliasing effect. Each trial was epoched with a $(-500,1000)$ ms time window, with respect to the image onset. Activity in the broad gamma band $(50-150 \mathrm{~Hz})$ was analyzed in the time-frequency (TF) domain by convolution with complex Morlet's wavelet. The broad gamma band is a typical feature of intracranial-EEG (iEEG) recordings in epileptic patients, and spatially and functionally more specific than the power modulation in other bands, or in the intracranial ERP (iERP; Vidal et al., 2010; Caruana et al., 2014a,b). Furthermore it is currently associated to neuronal population spiking activity (Manning et al., 2009; Ray and Maunsell, 2011), and to the BOLD signal as well (Logothetis et al., 2001; Lachaux et al., 2007). In line with our previous intracranial studies (Caruana et al., 2014a,b) gamma power was estimated for 10 adjacent non-overlapping frequency bands, each $10 \mathrm{~Hz}$ wide, and a divisive baseline correction was applied versus the prestimulus interval $(-500 / 0)$. The entire analysis pipeline was performed by integrating EEGLAB $^{\circledR}$ functions with home-made Matlab $^{\circledR}$ code.

\section{STATISTICAL ANALYSIS}

The analysis was performed on all the contacts located in BA6, according to their MNI coordinates. Forty-six contacts, out of 574 implanted ones, were located in different sections of the precentral gyrus; more specifically seven were located in the vPMC, 22 were located in the $\mathrm{APMC}$ and 17 were located in the medial parts of Brodmann Area 6 (mBA6; see Table 1 for the localization of the entrance points). To evaluate the presence of early visual responses in the precentral region we preliminarily calculated with a one-sample $t$-test the significance of gamma band power values versus a zero-mean distribution, using time bins of $20 \mathrm{~ms}$ each, in a $(-100,100) \mathrm{ms}$ time window. The analysis was applied to the six different conditions, separately. This pre-scanning was aimed to evaluate whether BA6 sites present any early response to the task regardless the between conditions differences. Given the lack of conventional analysis in sEEG we adopted this procedure from electrophysiological studies on single
Table 1 | Electrode entrance points.

\begin{tabular}{llll}
\hline \multicolumn{3}{c}{ Entrance points } \\
\hline Patient & Electrode & MNI & Hemisphere \\
\hline P1 & F & $-52.0,-0.8,46.9$ & $\mathrm{~L}$ \\
P1 & $\mathrm{K}$ & $-19.9,19.5,59.0$ & $\mathrm{~L}$ \\
P1 & $\mathrm{M}$ & $-31.0,-15.7,69.2$ & $\mathrm{~L}$ \\
P2 & $\mathrm{J}$ & $-16.7,21.1,58.4$ & $\mathrm{~L}$ \\
P2 & $\mathrm{L}$ & $-16.7,21.1,58.4$ & $\mathrm{~L}$ \\
P3 & $\mathrm{H}$ & $-58.9,3.4,32.4$ & $\mathrm{~L}$ \\
P3 & $\mathrm{M}$ & $-42.7,-8.9,59.4$ & $\mathrm{~L}$ \\
P4 & $\mathrm{M}$ & $48.1,-5.6,52.4$ & $\mathrm{R}$ \\
P5 & $\mathrm{M}$ & $-57.8,-1.3,36.5$ & $\mathrm{~L}$ \\
P6 & $\mathrm{N}$ & $-49.9,-1.1,48.5$ & $\mathrm{~L}$ \\
\hline
\end{tabular}

The MNI coordinates of the entrance points and the side of implantation of each electrode are shown.

unit recordings in the monkeys, as sEEG recordings resemble such technique concerning the inhomogeneous spatial sampling and the spatial selectivity of the activity. A similar approach on sEEG data was used by our group in the past (Caruana etal., 2014b). All contacts showing a significant gamma modulation in at least two consecutive time bins in any of the conditions were considered for more detailed analyses. More specifically, to evaluate whether the early visual response was modulated by the incongruent, congruent or neutral conditions, a repeated measures ANOVA was applied to each significant contact, considering CONDITION (congruent, incongruent, and neutral) and TIME [10 adjacent $20 \mathrm{~ms}$ time bins $(-100,100)]$ as factors, for both the contralateral and the ipsilateral responses. In order to account for the multiple comparisons issue, the p-threshold used to consider an ANOVA effect (either main effect or interaction) as significant was Bonferroni corrected dividing the standard $5 \%$ value for the number of carried out independent analyses, i.e., the amount of significant leads. For each significant interaction, post hoc analysis was conducted by means of a paired $t$-test.

A second analysis was aimed to evaluate the presence of a delayed response, during the action selection process. First we calculated with a one-sample $t$-test the significance of gamma band power values versus a zero-mean distribution, in a $(-500$, 1000) ms time window. As this time window was much larger, we decided to increase the single time bin size to avoid an overdetailing of a spreaded-over-time effect, using time bins of $100 \mathrm{~ms}$ each. The analysis was applied to the three conditions (congruent, incongruent, and neutral) requiring an ipsilateral response. Only the correct trials were analyzed. The same analysis was not applied to the trials requiring a contralateral response to avoid the risk of false positives due to the delayed motor responses in the incongruent trials. All contacts showing a significant gamma modulation in at least one time bin in one of the three conditions were considered task-related and considered for a more detailed analysis. More specifically, to evaluate whether the late response 
was modulated by the incongruent, congruent or neutral conditions, a repeated measures ANOVA was applied to each significant contact, considering CONDITION (congruent, incongruent, and neutral) and TIME [30 adjacent 50 ms time bins $(-500,1000)]$ as factors. For each significant interaction, post hoc analyses were performed by means of a paired $t$-test.

Finally, to evaluate whether the contacts showing a response during the action selection process were also involved in the action execution, we statistically compared their gamma band modulation during trials requiring a responses with the ipsilateral hand to the trials requiring a response with the contralateral hand, grouping together the three conditions. A repeated measures ANOVA was applied considering HAND (contralateral, ipsilateral) and TIME [30 adjacent $50 \mathrm{~ms}$ time bins $(-500,1000)$ ] as factors. ANOVA $p$-values were not corrected for the number of explored leads because, differently from scalp and non-invasive recordings, sEEG records local activity that is rather independent among different leads. In contrast, for each lead exhibiting a significant TIME *CONDITION interaction, we run a post hoc analysis by means of paired $t$-test.

\section{RESULTS}

\section{BEHAVIORAL STUDY}

We performed a repeated measures ANOVA on RTs measured during iEEG recording in both rightward and leftward responses, considering the SIDE (rightward vs. leftward) and the CONDITION (congruent, incongruent, and neutral) as factors. Wrong responses were discarded from the analysis. The analysis showed a clear influence of the factor condition on RT $[F(2,442)=52.232$, $p<0.0001]$, but we did not find any significant effect of side
$[F(1,221)=0.02318, p=0.87913]$. Post hoc comparisons shown that RTs were significantly longer in the incongruent condition $(449.6 \pm 8 \mathrm{~ms}$ and $443.2 \pm 8 \mathrm{~ms}$, for rightward and leftward, respectively) than in the congruent (401.5 $\pm 8 \mathrm{~ms}$ and $384.9 \pm 8 \mathrm{~ms}$ ) and neutral conditions $(394.7 \pm 8 \mathrm{~ms}$ and $404.4 \pm 9 \mathrm{~ms} ; p<0.0001)$. Furthermore, no significant differences were found between the rightward and leftward responses, within each experimental condition (see Figure 1, lower panel).

\section{SHORT LATENCY RESPONSES IN dPMC}

A preliminary analysis was aimed to evaluate a significant gamma modulation within the early $100 \mathrm{~ms}$ per each contact and condition, separately. This analysis showed that only seven contacts out of 46 had a significantly stronger gamma modulation in at least two consecutive time bins in at least one of the six conditions. All of them were located in the dPMC (see Figure 2, left panel, and Table 2), while the vPMC and the mBA6 did not show any early activation. The repeated measures ANOVA performed to these contacts in both the contralateral and the ipsilateral trials showed a significant effect of TIME for all the investigated contacts $(p<0.05$ Bonferroni corrected). In contrast, the effect of CONDITION, as well as the CONDITION $\times$ TIME interaction, were not significant in any of the investigated contacts.

\section{CHOICE-RELATED RESPONSES IN dPMC}

In the behavioral Eriksen flankers task the incongruent condition produces a delayed response, compared to the congruent and the neutral conditions, and the increase in reaction times is considered to reflects the interference between competing responses. Accordingly, the second set of analyses was aimed to evaluate

Table 2 | Results.

\begin{tabular}{|c|c|c|c|c|c|c|c|c|c|}
\hline \multicolumn{5}{|c|}{ Short latency responses } & \multicolumn{5}{|c|}{ Movement related responses } \\
\hline P1 & $F^{\prime} 10$ & $-32.7,-5.1,44.6$ & L & $\mathrm{dPMC}$ & $\mathrm{P} 1$ & M'1 & $-5.0,-13.8,54.9$ & $\mathrm{~L}$ & mBA6 \\
\hline P1 & M'6 & $-18.1,-18.2,69.1$ & L & mBA6 & $\mathrm{P} 1$ & M'2 & $-4.1,-16.4,56.6$ & $\mathrm{~L}$ & mBA6 \\
\hline P3 & $M^{\prime} 11$ & $-35.9,-7.8,54.6$ & L & $\mathrm{dPMC}$ & $\mathrm{P} 1$ & M'3 & $-5.8,-20.8,57.5$ & $\mathrm{~L}$ & mBA6 \\
\hline P3 & $M^{\prime} 12$ & $-42.3,-8.1,59.3$ & L & $\mathrm{dPMC}$ & $\mathrm{P} 1$ & $M^{\prime} 4$ & $-6.7,-22.0,55.2$ & $\mathrm{~L}$ & mBA6 \\
\hline P4 & M8 & $36.9,-7.3,53.8$ & $\mathrm{R}$ & $\mathrm{dPMC}$ & $\mathrm{P} 1$ & M’5 & $-15.9,-18.9,71.0$ & $\mathrm{~L}$ & mBA6 \\
\hline P4 & M9 & $42.6,-6.6,53.8$ & $\mathrm{R}$ & $\mathrm{dPMC}$ & $\mathrm{P} 1$ & M'6 & $-17.1,-18.3,68.6$ & $\mathrm{~L}$ & mBA6 \\
\hline \multirow[t]{4}{*}{ P4 } & M10 & $44.8,-7.3,51.2$ & $\mathrm{R}$ & $\mathrm{dPMC}$ & $\mathrm{P} 1$ & M'8 & $-26.1,-16.0,68.5$ & $\mathrm{~L}$ & mBA6 \\
\hline & & & & & P3 & $M^{\prime} 6$ & $-25.7,-1.4,47.2$ & $L$ & $\mathrm{dPMC}$ \\
\hline & & & & & P3 & M'7 & $-28.1,-1.2,48.0$ & $\mathrm{~L}$ & $\mathrm{dPMC}$ \\
\hline & \multicolumn{4}{|c|}{ Choice related responses } & P3 & M'9 & $-31.7,-4.7,45.2$ & $\mathrm{~L}$ & $\mathrm{dPMC}$ \\
\hline P3 & $M^{\prime} 6$ & $-26.1,-1.7,47.2$ & $\mathrm{~L}$ & $\mathrm{dPMC}$ & P3 & $M^{\prime} 10$ & $-34.1,-8.3,50.7$ & $\mathrm{~L}$ & $\mathrm{dPMC}$ \\
\hline P3 & $M^{\prime} 7$ & $-28.8,-1.5,48.0$ & $\mathrm{~L}$ & $\mathrm{dPMC}$ & P3 & $M^{\prime} 11$ & $-35.3,-8.3,54.5$ & $L$ & $\mathrm{dPMC}$ \\
\hline P3 & $M^{\prime} 9$ & $-31.7,-4.7,45.2$ & $\mathrm{~L}$ & $\mathrm{dPMC}$ & P3 & $M^{\prime} 12$ & $-41.2,-7.1,58.5$ & $\mathrm{~L}$ & $\mathrm{dPMC}$ \\
\hline P3 & $M^{\prime} 10$ & $-35.2,-7.9,51.6$ & $\mathrm{~L}$ & $\mathrm{dPMC}$ & P3 & $M^{\prime} 13$ & $-42.7,-8.9,59.4$ & $\mathrm{~L}$ & $\mathrm{dPMC}$ \\
\hline P3 & $M^{\prime} 11$ & $-35.9,-7.8,54.6$ & $\mathrm{~L}$ & $\mathrm{dPMC}$ & P4 & M11 & $46.3,-4.1,51.8$ & $\mathrm{R}$ & $\mathrm{dPMC}$ \\
\hline P3 & $M^{\prime} 12$ & $-42.3,-8.1,59.3$ & $\mathrm{~L}$ & $\mathrm{dPMC}$ & P5 & $M^{\prime} 13$ & $-53.5,0.5,35.9$ & $L$ & VPMC \\
\hline P3 & $M^{\prime} 13$ & $-42.7,-8.9,59.4$ & $\mathrm{~L}$ & $\mathrm{dPMC}$ & P5 & $M^{\prime} 14$ & $-56.6,-0.6,37.3$ & $\mathrm{~L}$ & VPMC \\
\hline
\end{tabular}

The MNI coordinates of the significant sites, the side of implantation of each electrode, and its anatomical region, are shown for the three main statistical analyses. 

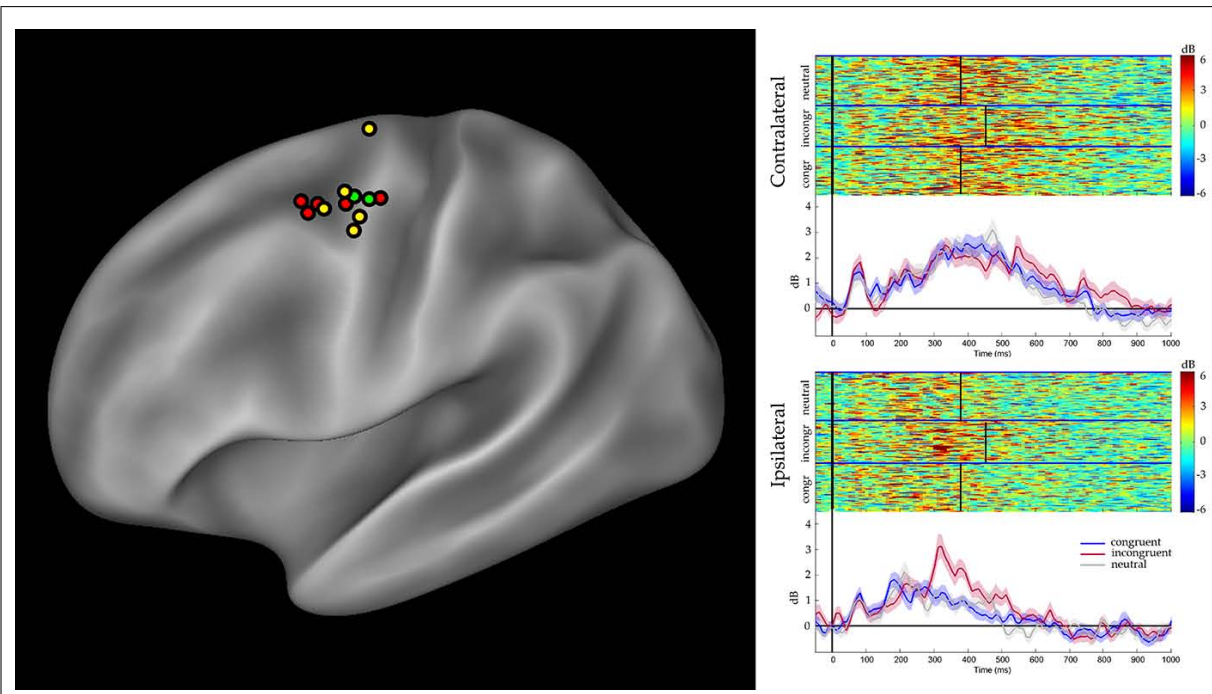

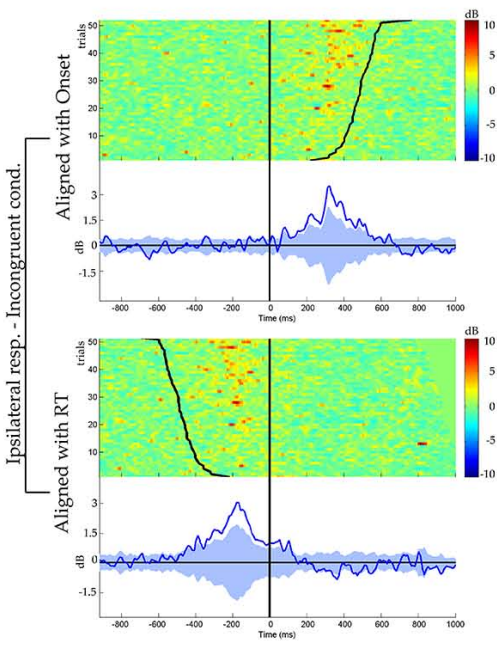

FIGURE 2 | (Left panel) Sites showing significant unspecific, shortlatency activations according to the gamma band reactivity within 100 ms after stimulus onset, are shown in yellow. Choice-related sites, showing significant activation during the incongruent trials, are shown in red. Furthermore, sites showing both effect are shown in green. Results are plotted on a template (Caret ${ }^{\circledR}$ ) according to their MNI coordinates. (Central panel) Results from a representative $d$ premotor cortex (PMC) site are shown (P3, $\left.M^{\prime} 12\right)$. Figure shows the gamma modulation during the congruent, incongruent, and neutral trials requiring a response with the hand contralateral (top) and ipsilateral (bottom) to the implanted hemisphere. Data are aligned with the stimulus onset. The average reaction time for the three conditions is shown at the single trial level (black vertical lines). The short-latency activations is clear in both contralateral- and ipsilateralresponse trials, in the three conditions. In the contralateral-response trials the activity is prolonged after the response, while in the ipsilateral-response trials is suppressed before the response. The greater activity during the incongruent conditions is visible in the ipsilateral-response trials. (Right panel) The same dataset, showing a representative dPMC site (P3, $\left.M^{\prime} 12\right)$ during the incongruent condition requiring a response with the ipsilateral hand, aligned to both the onset of the stimulus (upper part) and the onset of the response (lower panel). For each selected alignment, the other event is indicated by a curved line. how action selection processes are accommodated in the human motor system, and more specifically to assess whether the decision to respond (or to inhibit a response) affected the gamma band in the recorded contacts. As a preliminary analysis we selected the task-related contacts by means of a $t$-test applied to the congruent, incongruent, and neutral conditions requiring a response with the hand ipsilateral to the implanted hemisphere. Trials with errors or with a delayed response $(\mathrm{RT}>1 \mathrm{~s})$ were discarded. Results showed that 26 out of 46 contacts showed a significant response. Fourteen contacts were located in the dPMC, 10 in the mBA6 and 2 in the vPMC. The repeated measures ANOVA performed on these contacts showed a significant effect of TIME $(p<0.001$ Bonferroni corrected) in all the investigated contacts, and a significant CONDITION*TIME interaction in 7 out of 26 contacts ( $p<0.05$ Bonferroni corrected; see Figure 2 and Table 2). Post hoc comparison showed a significant increase of gamma modulation in the incongruent vs. the other two conditions. All the significant contacts were located in the left dPMC.

\section{MOVEMENT-RELATED RESPONSES}

Finally we evaluated whether the contacts that measured activity during action selection also measured activity during action execution. To this purpose, we grouped together all the responses according to whether they required a contralateral or an ipsilateral response, with respect to the recording site. The ANOVA showed a significant CONDITION*TIME $(p<0.05$ Bonferroni corrected) interaction in 17 out of 46 contacts (eight in dPMC, two in vPMC, and seven in mBA6; see Table 2). Post hoc comparison showed that $\mathrm{APMC}$ and vPMC sites showed a significantly prolonged activity during the contralateral trials, starting around $400 \mathrm{~ms}$ (i.e., while the subject were giving their manual response), thus suggesting a role of these regions in movement execution.

In contrast mBA6 sites showed a significantly stronger activity during trials requiring a response with the ipsilateral hand, and much earlier as compared to the other regions, that is, from 150 to $300 \mathrm{~ms}$, thus suggesting an involvement of mBA6 in the inhibition of the unrequested movement (see Figure 3).

\section{DISCUSSION}

In the present study we intracranially recorded from the $\mathrm{APMC}$, vPMC, and mBA6 during a bimanual version of the classic Eriksen flankers test (Eriksen and Eriksen, 1974). Only the dPMC appeared to play a specific role in action selection and showed a complex temporal pattern of response in the time window between the onset of the stimulus and the action execution. This complex response included (1) an early and unspecific activation, with a short latency within $100 \mathrm{~ms}$ from the onset of the stimulus; (2) a second activation, showing a modulation depending on the experimental condition, possibly reflecting an action selection process, and (3) a movement-related prolonged modulation when the response was given with the hand contralateral to the recording site. 


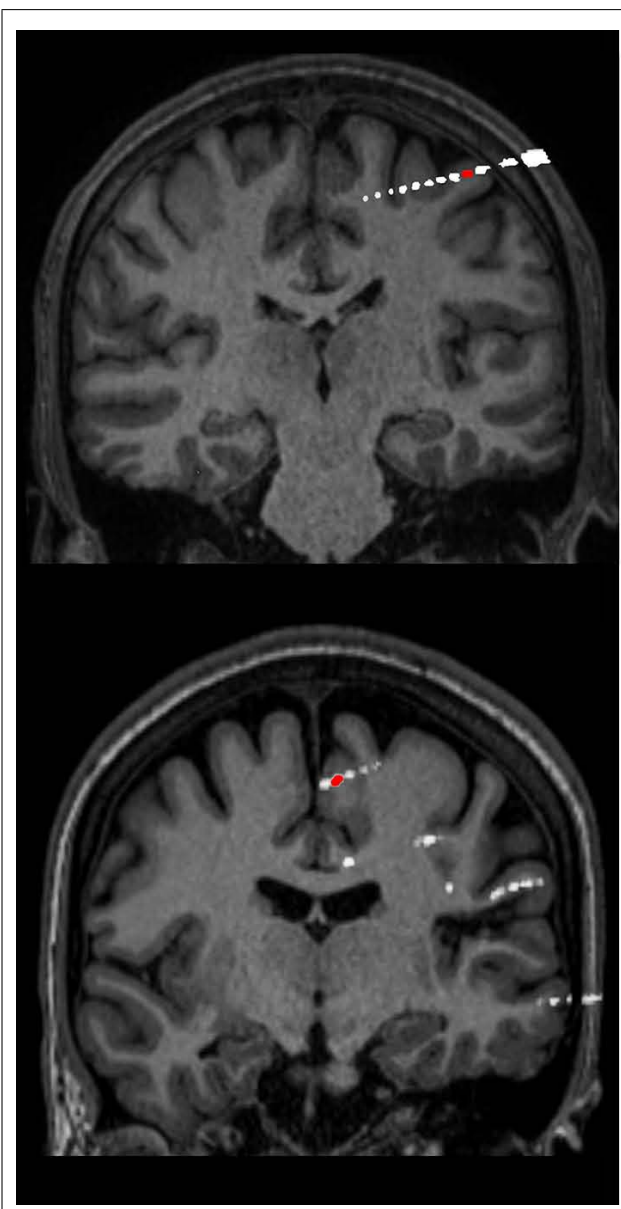

FIGURE 3 | Representative recording from a dPMC (top) and a mBA6 (bottom) sites. (Left panel) The exact localization of the recording site is shown by the coregistration of the individual MRI with postimplantation CT. (Central panel) Gamma activity recorded from the contacts in red. Images show the statistical comparison between trials from the three condition pooled together, and requiring a contralateral vs. ipsilateral response in a $-500,1000$ time window, in 30 adjacent time bins of $50 \mathrm{~ms}$. Responses are aligned on the stimulus onset. Red asterisks indicate the significant post hoc comparisons. (Right panel) Time-frequency plots of the gamma activity $(50-150 \mathrm{~Hz})$ showing the frequency profile of the same electrodes, during contralateral and ipsilateral responses, in a $-500,1000$ time window.
The very first question concerns the nature of the first early activation of the dPMC. At a first glance, the three distinct neuronal events appear to be a human counterpart of the signal-related, setrelated and movement-related activity described in the monkey dPMC neurons (Wise, 1985; Boussaoud and Wise, 1993). However, in contrast to the classic signal-related neurons, firing at the appearance of the instruction stimulus and differently modulated according to the instructed movement (Kalaska et al., 1997; Wise et al., 1997), the early modulation we have found is unspecific to the type of stimulus. Furthermore, its latency is too short to be a human counterpart of a signal-related activity, as response-specific processing in the monkey and human motor system starts from $150 \mathrm{~ms}$ after stimulus onset (Thorpe et al., 1996; Thut et al., 2000; Ledberg et al., 2007). In contrast, our results are well in line with previous studies describing similar unspecific responses characterized by very short latencies $(<100 \mathrm{~ms})$, as early as in the striate cortex, in both humans and monkeys (Foxe and Simpson, 2002; Ledberg et al., 2007). Unspecific early stimulus-evoked responses followed by more complex stimulus-specific processing, have been also described at the single neuronal level in the monkey dPMC and FEF during action selection tasks (Schall and Bichot, 1998; Cisek and Kalaska, 2005; see also Crammond and Kalaska, 1994). To date, the best explanation for these responses is that salient stimuli automatically attract motor attention before the preparation of the appropriate motor response, and suggests that a recruitment of the motor system by salient stimuli occurs well before its involvement in action planning. This early activation is posited to start an action specification and selection process in which action options are implemented as sensorimotor loops that are biased by several processes in, among others, prefrontal areas and sensorimotor loops (Cisek, 2007). This model predicts a phase in which action options are not yet specified or differentiated, but already present in the motor system. The early and undifferentiated response we report here fits this model.

The unspecific response was followed by a second peak preceding the movement onset. This activation was also found in most vPMC and mBA6 sites, but only in the dPMC this activity was modulated by the congruent, incongruent or neutral condition, 
showing a stronger response following the presentation of incongruent stimuli (see Figure 2, right panel). This data provide a mechanistic insight to the role played by the dPMC in the Eriksen flankers task, and in action selection more in general. It is known that flanking the central directional cue with incongruent directional stimuli elicits competing responses but, beside some indirect evidence on the involvement of the fronto-parietal circuits (Coulthard et al., 2008; Michelet et al., 2010; see also Cisek, 2008), little is known on the specific regions of the human brain in which this competition is solved. In contrast, electrophysiological studies on the monkey motor system provided mechanistic evidences to the role of the APMC in action selection. Single unit recording during reach-selection tasks showed that, in the period of uncertainty between two opposite potential actions, the dPMC reflects both responses, while only after the information for selecting one action became available the representation of the chosen direction became strengthened (Cisek and Kalaska, 2005; see also Crammond and Kalaska, 1994; Gail et al., 2009). A similar multiple activation has been also shown in the adjacent FEF region during visual search tasks, showing an early modulation to all salient stimuli and a later one reflecting only the final selected target (Schall and Bichot, 1998). Similar mechanisms were also described in the posterior parietal cortex, in particular in the parietal reach region (PRR) connected with the $\mathrm{APMC}$, and in the lateral intraparietal sulcus (LIP) connected with the FEF (see Andersen and Cui, 2009 for a review). They may be objections that in these studies the monkeys were intensively trained to accomplish experimental tasks aimed to map perceptual decisions onto motor outputs and, as a consequence, their results could be affected by this intense training (see Kubanek and Kaplan, 2012). Our data shows that the findings in monkeys cannot be attributed to the intensive training in monkeys. Participants in our study only received a very short training session of less than two minutes. It remains to be seen whether these findings are induced by the forced-choice paradigm and can therefore be best understood as "task-set related" (Sakai, 2008), or whether the same principles underlie freely chosen actions as well.

According to the affordance competition hypotheses, whenever multiple activations, instructing opposite responses, appear simultaneously within a given region, they compete against each other for further processing (Cisek, 2007). The exact mechanisms for action selection, however, are still unknown. For example, the correct response could be enhanced until it crosses a threshold, or the wrong response could be suppressed until it drops below a certain threshold (or some delicate interplay between these two mechanisms). Although our study cannot provide concluding evidence, it does suggest that at least part of action selection is based on inhibiting the incorrect response.

Competition is assumed to be strongest upon incongruent trials, as concomitant rightward and leftward arrows automatically enhance the activity in favor of both left and right responses. Additionally, activity related to suppressing the wrong option is expected to be highest on the ipsilateral side, as this is the side that is not supposed to generate a response. Figure 2, left panel, clearly shows a significantly higher activation for incongruent trials at the ipsilateral side between 300 and $450 \mathrm{~ms}$, being the phase right before the response.
In Figure 2, right panel, the same dataset from incongruent trials requiring a response with the hand ipsilateral to the recording site, is aligned to both the onset of the stimulus (upper part) and onset of the response (lower panel). The significant drop of activity before the movement onset, clear in the second alignment, suggests that the behavioral response depends on the suppression of the ipsilateral dPMC, and not on the increase of the contralateral dPMC (see left panel). So it seems that the competition between the two dPMC is won by the contralateral dPMC when the ipsilateral $\mathrm{dPMC}$ retreats. It is unlikely that this higher activation is caused by "general conflict," rather than suppression, as conflict is expected to have an equal impact on both hemispheres (i.e., conflict is not related to the subsequent response), while our data shows greater activity only on the ipsilateral side. However, further experiments, including recordings in the right $\mathrm{PMC}$ are needed to substantiate this hypothesis.

We have shown that the APMC shows a short latency activation, suggesting a relatively direct visual input. From monkey physiological findings it is known that area F2 - which is supposed to be the monkey counterpart of the caudal dPMC - receives visual input through MIP (medial bank of the intraparietal sulcus), and V6A (Matelli et al., 1998). The latter - which is suggested to have a human homolog (Pitzalis et al., 2013) - is of special interest here, as this parieto-occipital area connects to V2, V3, and V4, as well as V1 through V6 (Passarelli et al., 2011) and provides therefore relatively direct visual input. Moreover, about half of the neurons in V6A discharge in response to visual stimuli (Galletti et al., 1999), while the response in MIP seems to be stronger related to reaching movements.

Medial BA6 sites showed a significantly stronger activity during trials requiring a response with the ipsilateral hand after the first undifferentiated activation in $\mathrm{APMC}$, suggesting an involvement of this region in the inhibition of the wrong response (see Figure 3 ). This inhibitory role is in line with existing findings (Mars et al., 2009).

The vPMC shows only late onset activation that lasts well into the execution phase. However, since we have data of only few recording sites in vPMC, it is hard to draw firm conclusions from this.

If these findings are interpreted in terms of action selection through competition, it seems that the process of action specification start at the APMC. The medial part of BA6 are subsequently involved in selecting the appropriate action, while vPMC is primarily involved in executing the action. In line with this model, previous intracranial recording from patients engaged in the Eriksen flankers task shows that incongruent trials correlate with a reduction of the theta phase alignment in the subthalamic nucleus (Zavala et al., 2013), that is indirectly connected with the caudal dPMC in the monkey (Saga et al., 2011). Together these findings suggest that the Erikson Flankers task evokes a response competition in humans similar to the ones found upon spatial color cues in monkeys. Of course, many other areas might be involved in the specification and selection processes, and based on this data set we cannot even conclude that these areas provide the backbone of the action specification and selection mechanism. More studies including more patients as well as more recording sites are needed. 
While probably every empirical finding can be accommodated in both the classical and the embodied or enactivist approaches, we believe that these findings are much more in line with the latter frameworks. In principle, the classical framework would not predict an early and undifferentiated response in the motor cortices, as the motor system need not be active until a decision by cognitive processes have been made. That being said, it is possible to account for this early activation within this framework. One could for example argue that this is a non-functional side-effect of expecting $a$ stimulus, while not knowing in advance which stimulus. Due to the instructions to respond as fast as possible, the motor system is brought to a heightened state of activation, and the mere appearance of the stimulus might be enough to cause a stir in the motor system. But it should be noted that this is a post hoc adjustment of the framework, and one would expect this to be a more general effect also present at vPMC and the mBA6. On the other hand, if action options are evaluated in the sensorimotor cortex, as suggested by the affordance competition hypothesis, the early activation is an essential component of the model (cf. also van Dijk et al.,'s 2008 "traffic regulator metaphor" for embodied embedded neuroscience). As soon as a stimulus appears, action options are activated as a sensorimotor loop. These loops are subsequently evaluated, biased, and selected predicting an early undifferentiated response, and a later differentiated response exactly as we reported. The fact that the APMC shows two subsequent activations, one undifferentiated, one condition-dependent, strongly suggests a processing loop, rather than successive cognitive stages.

A possible objection to our interpretation of this result and the model of Cisek (2007) in enactivist terms (Varela et al., 1991; Hutto and Myin, 2013) is that merely replacing one representation of a to be planned action with two representations we still assume representationalism. However, the parallel action options should emphatically not be interpreted as representations, but rather as dynamic processes (Chemero, 2009). The options are not discrete states with fixed content, but develop over time in complex interaction with various brain structures as well as with the skeletomuscular system (Schurger and Uithol, in press). This dynamic nature cannot be accounted for using a representational approach. In all, the data discussed above suggest that the PMC accommodates or is part of a complex network in which action options are specified and selected in parallel. The nature and the pathways that favor one option over the other remains to be investigated, but our findings suggest that inhibition of the incorrect response, specifically by the medial part of BA6 and the dPMC plays an important role.

\section{ACKNOWLEDGMENTS}

We thank Elena Borra for most valuable comments on an earlier version of the manuscript. This work was supported by ERC Advanced Grant CogSystem. Sebo Uithol was supported by the EU grant "Towards an Embodied Science of Intersubjectivity" (TESIS, FP7-PEOPLE-2010-ITN, 264828).

\section{REFERENCES}

Andersen, R. A., and Cui, H. (2009). Intention, action planning, and decision making in parietal-frontal circuits. Neuron 63, 568-583. doi: 10.1016/j.neuron.2009.08.028
Boussaoud, D., and Wise, S. P. (1993). Primate frontal cortex: effects of stimulus and movement. Exp. Brain Res. 95, 28-40. doi: 10.1007/BF00229651

Caruana, F., Cantalupo, G., Russo, G. L., Mai, R., Sartori, I., and Avanzini, P. (2014a). Human cortical activity evoked by gaze shift observation: an intracranial EEG study. Hum. Brain Mapp. 35, 1515-1528. doi: 10.1002/hbm.22270

Caruana, F., Sartori, I., Lo Russo, G., and Avanzini, P. (2014b). Sequencing biological and physical events affects specific frequency bands within the human premotor cortex: an intracerebral EEG study. PLOS ONE 9:e86384. doi: 10.1371/journal.pone.0086384

Chemero, A. (2009). Radical Embodied Cognitive Science. Cambridge, MA: MIT Press.

Cisek, P. (2007). Cortical mechanisms of action selection: the affordance competition hypothesis. Philos. Trans. R. Soc. Lond. B Biol. Sci. 362, 1585-1599 doi: 10.1098/rstb.2007.2054

Cisek, P. (2008). A remarkable facilitating effect of parietal damage. Neuron 58, 7-9. doi: 10.1016/j.neuron.2008.03.025

Cisek, P., and Kalaska, J. F. (2005). Neural correlates of reaching decisions in dorsal premotor cortex: specification of multiple direction choices and final selection of action. Neuron 45, 801-814. doi: 10.1016/j.neuron.2005.01.027

Cisek, P., and Kalaska, J. F. (2010). Neural mechanisms for interacting with a world full of action choices. Annu. Rev. Neurosci. 33, 269-298. doi: 10.1146/annurev.neuro.051508.135409

Coulthard, E. J., Nachev, P., and Husain, M. (2008). Control over conflict during movement preparation: role of posterior parietal cortex. Neuron 58, 144-157. doi: 10.1016/j.neuron.2008.02.009

Crammond, D. J., and Kalaska, J. F. (1994). Modulation of preparatory neuronal activity in dorsal premotor cortex due to stimulus-response compatibility. J. Neurophysiol. 71, 1281-1284.

Engel, A. K., Maye, A., Kurthen, M., and König, P. (2013). Where's the action? The pragmatic turn in cognitive science. Trends Cogn. Sci. 17, 202-209. doi: 10.1016/j.tics.2013.03.006

Eriksen, B. A., and Eriksen, C. W. (1974). Effects of noise letters upon the identification of a target letter in a nonsearch task. Percept. Psychophys. 16, 143-149. doi: 10.3758/BF03203267

Fodor, J. A. (1983). Modularity of Mind. Cambridge, MA: MIT Press.

Foxe, J. J., and Simpson, G. V. (2002). Flow of activation from V1 to frontal cortex in humans. A framework for defining "early" visual processing. Exp. Brain Res. 142, 139-150. doi: 10.1007/s00221-001-0906-7

Gail, A., Klaes, C., and Westendorff, S. (2009). Implementation of spatial transformation rules for goal-directed reaching via gain modulation in monkey parietal and premotor cortex. J. Neurosci. 29, 9490-9499. doi: 10.1523/JNEUROSCI.109509.2009

Galletti, C., Fattori, P., Kutz, D. F., and Gamberini, M. (1999). Brain location and visual topography of cortical area V6A in the macaque monkey. Eur. J. Neurosci. 11, 575-582. doi: 10.1046/j.1460-9568.1999.00467.x

Hutto, D., and Myin, E. (2013). Radicalizing Enactivism: Basic Minds Without Content. Cambridge, MA: MIT Press.

Kalaska, J. F., Scott, S. H., Cisek, P., and Sergio, L. E. (1997). Cortical control of reaching movements. Curr. Opin. Neurobiol. 7, 849-859. doi: 10.1016/S09594388(97)80146-8

Kubanek, J., and Kaplan, D. M. (2012). Evidence for a decision variable in the human motor system. J. Neurosci. 32, 8110-8111. doi: 10.1523/JNEUROSCI.181312.2012

Lachaux, J. P., Fonlupt, P., Kahane, P., Minotti, L., Hoffmann, D., Bertrand, O., et al. (2007). Relationship between task-related gamma oscillations and BOLD signal: new insights from combined fMRI and intracranial EEG. Hum. Brain Mapp. 28, 1368-1375. doi: 10.1002/hbm.20352

Ledberg, A., Bressler, S. L., Ding, M., Coppola, R., and Nakamura, R. (2007). Largescale visuomotor integration in the cerebral cortex. Cereb. Cortex 17, 44-62. doi: 10.1093/cercor/bhj123

Logothetis, N. K., Paulsm J., Augath, M., Trinath, T., and Oeltermann, A. (2001). Neurophysiological investigation of the basis of the fMRI signal. Nature 412, 150-157. doi: 10.1038/35084005

Manning, J. R., Jacobs, J., Fried, I., and Kahana, M. J. (2009). Broadband shifts in local field potential power spectra are correlated with single-neuron spiking in humans. J. Neurosci. 29, 13613-13620. doi: 10.1523/JNEUROSCI.2041-09.2009

Mars, R. B., Klein, M. C., Neubert, F. X., Olivier, E., Buch, E. R., Boorman, E. D., et al. (2009). Short-latency influence of medial frontal cortex on primary motor 
cortex during action selection under conflict. J. Neurosci. 29, 6926-6931. doi: 10.1523/JNEUROSCI.1396-09.2009

Matelli, M., Govoni, P., Galletti, C., Kutz, D. F., and Luppino, G. (1998). Superior area 6 afferents from the superior parietal lobule in the macaque monkey. J. Comp. Neurol. 402, 327-352. doi: 10.1002/(SICI)1096-9861(19981221)402:3<327::AIDCNE4>3.0.CO;2-Z

Michelet, T., Duncan, G. H., and Cisek, P. (2010). Response competition in the primary motor cortex: corticospinal excitability reflects response replacement during simple decisions. J. Neurophysiol. 104, 119-127. doi: 10.1152/jn.00819.2009

Miller, G. A., Galanter, E., and Pribram, K. H. (1960). Plans and the Structure of Behavior. New York: Holt, Rinehart and Winston. doi: 10.1037/10039-000

Passarelli, L., Rosa, M. G. P., Gamberini, M., Bakola, S., Burman, K. J., Fattori, P., et al. (2011). Cortical connections of area V6Av in the macaque: a visualinput node to the eye/hand coordination system. J. Neurosci. 31, 1790-1801. doi: 10.1523/JNEUROSCI.4784-10.2011

Pitzalis, S., Sereno, M. I., Committeri, G., Fattori, P., Galati, G., Tosoni, A., et al. (2013). The human homologue of macaque area V6A. Neuroimage 82, 517-530 doi: 10.1016/j.neuroimage.2013.06.026

Ray, S., and Maunsell, J. H. (2011). Different origins of gamma rhythm and high gamma activity in macaque visual cortex. PLoS Biol. 9:e1000610. doi: 10.1371/journal.pbio.1000610

Saga, Y., Hirata, Y., Takahara, D., Inoue, K., Miyachi, S., Nambu, A., et al. (2011). Origins of multisynaptic projections from the basal ganglia to rostrocaudally distinct sectors of the dorsal premotor area in macaques. Eur. J. Neurosci. 33, 285-297. doi: 10.1111/j.1460-9568.2010.07492.x

Sakai, K. (2008). Task set and prefrontal cortex. Annu. Rev. Neurosci. 31, 219-245. doi: 10.1146/annurev.neuro.31.060407.125642

Schall, J. D., and Bichot, N. P. (1998). Neural correlates of visual and motor decision processes. Curr. Opin. Neurobiol. 8, 211-217. doi: 10.1016/S0959-4388(98) 80142-6

Schurger, A. A., and Uithol, S. (in press). Nowhere and everywhere: the causal origin of voluntary action. Rev. Philos. Psychol.

Thorpe, S., Fize, D., and Marlot, C. (1996). Speed of processing in the human visual system. Nature 381, 520-522. doi: 10.1038/381520a0

Thut, G., Hauert, C. A., Blanke, O., Morand, S., Seeck, M., Gonzalez, S. L., et al. (2000). Visually induced activity in human frontal motor areas during simple visuomotor performance. Neuroreport 11, 2843-2848. doi: 10.1097/00001756200009110-00004

Uithol, S., Burnston, D., and Haselager, W. F. G. (2014). Why we may not find intentions in the brain. Neuropsychologia 56, 129-139. doi: 10.1016/j.neuropsychologia.2014.01.010
Uithol, S., van Rooij, I., Bekkering, H., and Haselager, W. F. G. (2012). Hierarchies in action and motor control. J. Cogn. Neurosci. 24, 1077-1086. doi: 10.1162/jocn_a_00204

van Dijk, J., Kerkhofs, R., van Rooij, I., and Haselager, W. F. G. (2008). Can there be such a thing as embodied embedded cognitive neuroscience? Theory Psychol. 18:297. doi: 10.1177/0959354308089787

Varela, F. J., Thompson, E., and Rosch, E. (1991). The Embodied Mind: Cognitive Science and Human Experience. Cambridge, MA: MIT Press.

Vidal, J. R., Ossandón, T., Jerbi, K., Dalal, S. S., Minotti, L., Ryvlin, P., et al. (2010). Category-specific visual responses: an intracranial study comparing gamma, beta, alpha, and ERP response selectivity. Front. Hum. Neurosci. 4:195. doi: 10.3389/fnhum.2010.00195

Wise, S. P. (1985). The primate premotor cortex: past, present, and preparatory. Annu. Rev. Neurosci. 8, 1-19. doi: 10.1146/annurev.ne.08.030185. 000245

Wise, S. P., Boussaoud, D., Johnson, P. B., and Caminiti, R. (1997). Premotor and parietal cortex: corticocortical connectivity and combinatorial computations. Annu. Rev. Neurosci. 20, 25-42. doi: 10.1146/annurev.neuro. 20.1 .25

Zavala, B., Brittain, J. S., Jenkinson, N., Ashkan, K., Foltynie, T., Limousin, P., et al. (2013). Subthalamic nucleus local field potential activity during the Eriksen flanker task reveals a novel role for theta phase during conflict monitoring. J. Neurosci. 33, 14758-14766. doi: 10.1523/JNEUROSCI.103613.2013

Conflict of Interest Statement: The authors declare that the research was conducted in the absence of any commercial or financial relationships that could be construed as a potential conflict of interest.

Received: 15 May 2014; accepted: 11 August 2014; published online: 26 August 2014. Citation: Caruana F, Uithol S, Cantalupo G, Sartori I, Lo Russo G and Avanzini P (2014) How action selection can be embodied: intracranial gamma band recording shows response competition during the Eriksen flankers test. Front. Hum. Neurosci. 8:668. doi: 10.3389/fnhum.2014.00668

This article was submitted to the journal Frontiers in Human Neuroscience.

Copyright (c) 2014 Caruana, Uithol, Cantalupo, Sartori, Lo Russo and Avanzini. This is an open-access article distributed under the terms of the Creative Commons Attribution License (CC BY). The use, distribution or reproduction in other forums is permitted, provided the original author(s) or licensor are credited and that the original publication in this journal is cited, in accordance with accepted academic practice. No use, distribution or reproduction is permitted which does not comply with these terms. 